\title{
Overview a Quality-Scalable and Energy-Efficient Approach for Spectral Analysis of Heart Rate Variability
}

\author{
Aditi Malviya \\ M.Tech. Research Scholar \\ T.I.E.I.T \\ Bhopal
}

\author{
Neelesh Gupta \\ Assistant Professor, HOD \\ T.I.E.I.T \\ Bhopal
}

\author{
Neetu Sharma \\ Assistant Professor \\ T.I.E.I.T \\ Bhopal
}

\begin{abstract}
In this paper presenting bioelectrical signals which are spectrally analyzed for enabling energy- quality trade-offs, they are helpful in observing different health problems as those related with the rate of heart. To facilitate such tradeoffs, the signals which are processed earlier are expressed primarily in a beginning in which considerable components that hold mainly of the related information can be simply notable from the components that effect the output to a smaller amount. Such an arrangement permits the pruning of operations allied with the less important signal components primary to power savings with loss of minor quality as simply less useful parts are reduced under the certain requirements. This provides the patients normal and abnormalities using ECG waves.
\end{abstract}

Keywords

Spectral Analysis, Heart Rate, Energy-Efficient, Haar, PSA

\section{INTRODUCTION}

OUR modern society is now in danger by the developing healthcare delivery problem caused by the current demographic and way of living craze. On the one hand, the world's population is rapid aging resultant into a bigger prevalence of cardiac disorders. On the other contrary, our hectic and regularly unhealthy lifestyles are fueling the increase of the number of people innocently developing or living with chronic cardiovascular states for decades. ECTROCARDIOGRAM (ECG or EKG) is a tool of diagnosis used to measure human heart's electrical activity and presents in detail. Many threatening heart diseases cure by interpreting these diagnosis details. The current is diffused around the surface of the body. An ECG curve is obtained by the nerve response to the heart.

The current at the body surface will build a voltage drop, which is a couple of microvolt to millivolt with an impulse variation. This is very small amplitude of impulse, which needs pair of amplification in thousand times. A general ECG tracing of a regular heart rate (or cardiac cycle) comprise of a waves like P, T and a QRS complex. ECG's baseline voltage called as isoelectric line. Typically, the isoelectric line is calculated by preceding the $\mathrm{P}$ wave and following the $\mathrm{T}$ as isoelectric portion of tracing.

Electrical activity of the heart can be recorded at the surface of the body using an electrocardiogram. Therefore, the electro-cardio-gram (EKG) is simply an instrument of voltage measuring that uses equal to 12 different leads (electrodes) implemented on required areas of the body.

As a subject of fact, according to the Monitoring electrodes located on skin surface generally helps in sensing the heart's electrical activity.

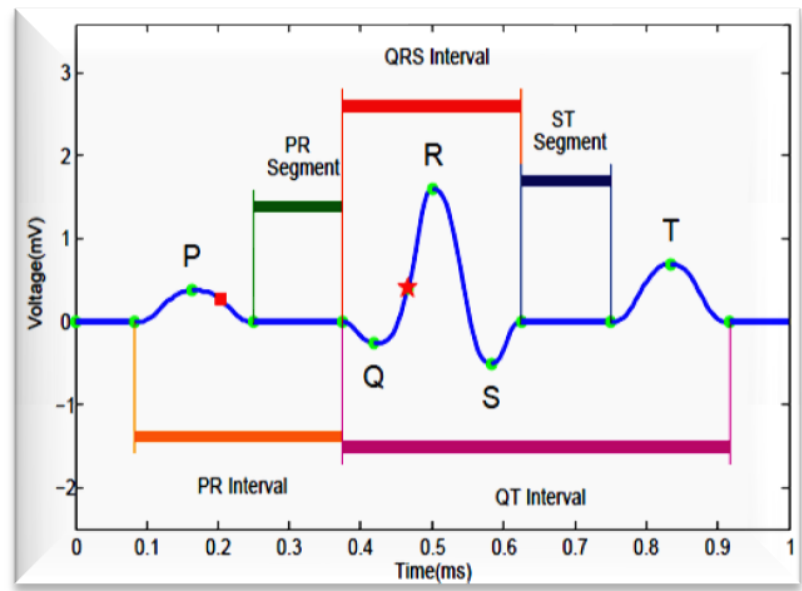

Fig:1 Ecg Signal

The ECG is simply used to record the electrical activity of heart. The changes in normal pattern of activity indicate numerous cardiac disorders. Cardiac cells are electrically polarized in normal conditions. The charge is negative on inner sides as compared to outer.

Electric current can be produced by depolarization of waves and can be detected by placing the electrodes on body surface.

These systems have various limitations as limited autonomy, large size and no or limited wireless connectivity. Recently, considerable industrial and academic interest is received by the use of realization of wireless-enabled low-power ECG monitors. The most important things to see of this research and progress efforts are: (1) Toumaz's Sensium Life Pebble TZ203082 [2], a very-small and very-low-power observer for heart rate, 5 days on a hearing aid battery; (2) Intel's ShimmerTM [3], a small wireless wearable sensor platform able to record and wirelessly transmit 3-lead ECG data as well as accelerometer, gyroscope and galvanic skin response information; (3) a minute wireless wearable sensor stand able to record and wirelessly pass on 3-lead ECG data as well as accelerometer, gyroscope and galvanic skin response information; (3) IMEC's wireless solo-lead bipolar ECG patch [4] used for ambulatory monitoring claiming over 10 days of monitoring on a $160 \mathrm{mAh} \mathrm{Li-ion} \mathrm{battery} \mathrm{(for} \mathrm{hidden}$ use conditions). The medical relevance of the primary structure is still being validated, as Toumaz aims to get extra than the system's so far established exact measurement of heart rate. The second system, that is base on profitable offthe-shelf workings such as the TI MSP430 microcontroller and the CC2420 radio chip-sets, operates on a Li-ion battery that supply energy of 1 Watt per hour . According to our practices in measurements, it is capable of supporting maximum 6.5-day single-lead raw ECG sensing and its local memory storage. When the raw ECG is run wirelessly with 
the use of the ultralow- power CC2420 in a link of perfect point-to-point through rejection wireless protocol overhead.

More significantly, this self sufficient figure will definitely significantly reduce under practical ambulatory monitor. At the end, IMEC very-low-power wireless biopotential sensor node achieves its improved autonomy thanks to a proprietary modified ultra-low-power analog read-out ASIC (signal acquisition, amplification and analog-to digital conversion), a proprietary very-low-power mega-wideband wireless transceiver, and more significantly, allotted signal processors that helps in pre-processing and compressing the sensed data using conventional techniques, this occurs to reduce the power-hungry wireless links airtime. Based on these premises, it is an increasingly thus, in terms of very-low-power keen digital processors and In this work, we look at a novel and capable approach based on the rising compressed sensing (CS) structure to deal with the challenges of very-low power set in compression of ECG signals. For further purposely, we compute the potential of the compressed sensing signal gaining/density pattern for low-difficulty power-proficient ECG compression, and demonstrate that it represent a competitive substitute to modern ECG compression answer in the framework of WBSN-base observe system. Since in the beginning days of seventies, ECG data compression has witness noteworthy advances fueled by, on the one side, the ever-increasing desire of doctor to store the enormous quantity of ECG data get in clinical apply, and on the other side, the emergence of portable tele-cardiology over bandwidth-limited public wireless networks. Until recently (and still practically today),"mobile" tele-cardiology however referred to wireless ECG communication from an ambulance or a patient's home to the hospital.

Heart rate variability (HRV) is a process of measurement (quantification) of middle autonomic drive (action) to the myocardium. It is depends upon a stability among drives of sympathetic and parasympathetic to myocardium. In difference to various conventional tests to estimate autonomic nervous system, Heart rate variability (HRV) examination stands as a non invasive technique of detecting an early autonomic mutilation of heart. A person considered have a good cardiac adaptability with high HRV, on the other hand lower HRV shows an unusual and insufficient adaptability of nervous system and has a high risk of numerous cardiovascular diseases.11. Heart rate variability (HRV) is also a good forecaster for risk occurrence and progression of crucial coronary atherosclerosis. Evrengul demonstrated decreased Heart rate variability (HRV) in patients through RA. Hence, evaluation of HRV has a main role in identifying the patients with RA who are at high danger of life dangerous cardiac events. The utility of HRV evaluation can be expanded to a timely analysis of altered autonomic role status linked with enlarged mortality in patients with RA. The existing literature shows no study work that has accessible HRV data in Indian patients with disease rheumatoid arthritis and its connection with disease doings.

\section{OVERVIEW}

Hossein Mamaghanian et al," Compressed Sensing for RealTime Energy-Efficient ECG Compression on Wireless Body Sensor Nodes", This paper author proposed a complete system-level comparison between a fresh CS-base and the modern DWT-based embed ECG compression algorithms for monitoring. As expected, non adaptive CS-based compression algorithm was found to exhibit inferior compression presentation judge against to its signal-adaptive DWT based equivalent for a given rebuild signal quality examination.
This paper presented results, however, were obtained using the default basis pursuit de-noise algorithm for sparse reconstruction signal, where no effort has been prepared to utilize the extreme structured nature of the ECG signal. Regardless of its lesser compression performance, CS-based compression algorithm was found to exhibit the best overall energy efficiency due to its lower complexity part and lesser CPU execution time for Shimmer WBSN stage. This paper results validate the appropriateness of CS for real-time powerproficient ECG compression on supply-constrained WBSN modes. More importantly, they suggest the relevance of pursuing an "analog CS" execution for the joint model and compression of ECG in the perspective of WBSN applications.[1].

Fabien Massé ,"Miniaturized wireless ECG monitor for realtime detection of epileptic seizures", In this paper author proposed recent advances in miniaturization of ultra-low power components allow for more intelligent wearable signal health monitors. The development and evaluation of a wireless wearable electrocardiogram (ECG) signal monitor to detect epileptic seizures from changes in the cardiac rhythm is described on paper. Authors are analyzed ECG data by embedded algorithms: a robust beat-detection algorithm combined with a real-time epileptic seizure detector. In its current implementation, the proposed prototype is $52 \times 36 \times$ $15 \mathrm{~mm}^{3}$, and has autonomy of one day $24^{\times} 7$. Based on data collected on the first three epilepsy patients, preliminary clinical results are provided by algorithm. Wireless, miniaturized and comfortable, this paper proposed new method opens new perspectives for health monitoring.

Yazicioglu, et al," Ultra-low-power wearable biopotential sensor nodes", this paper author discusses very-low-power wireless sensor nodes proposed for wearable bio-potential monitoring algorithm. Specific focus is specified to mixedsignal design approach and their impacts on the whole structure power dissipation, like of deals-offs in the power dissipation among analog front-end and digital signal processing are specified as well. It is demonstrated how signal filtering can reduce more the inner power utilization of a node with suitable speed. Kinds of power saving practices are essential as real-life tests of modified wireless ECG patch disclose the need for artifact recognition and alteration of real life. The power utilization of such supplementary features has to come from power reserves elsewhere in the system as on the whole power budget cannot boost.

Maistrou," Implicit comparison of accuracy of heart rate variability spectral measures estimated via heart rate and heart period signals", In this paper a general idea of existing signals for estimation of HRV spectral domain actions is made. The analysis of functional dependency has been done among heart rate and heart period signals.

Shao-Yen Tseng et al, "An effective heart rate variability processor design based on algorithm of time-frequency analysis using windowed Lomb periodogram" In this paper, author presented HRV algorithm. This system author has been implemented in hardware as an HRV processor and verified on FPGA. Simulations demonstrate that the proposed Lomb TFD is capable to achieve enhanced frequency resolution than short period Fourier transform algorithm of the similar hardware size. The proposed system is appropriate for moveable monitoring devices and as a biomedical signal processor as part of system-on-chip (SOC) design. 


\section{MODULE}

\subsection{ECG}

An electrocardiogram (EKG or ECG) is a testing system that checks for problems with the electrical activity of your heart. An EKG translates the heart's electrical activity into line tracings on paper as part of plot. Most ECGs are performed for diagnostic or research purposes on human heart rates, but may also be performed on animals, usually for diagnosis of heart abnormalities or research. The spikes and dips in the line tracings plot are called waves. The heart is a muscular pump made up of four chambers. An ECG is used to measure the heart's electrical conduction system and it picks up electrical impulses generated by the polarization and depolarization of cardiac tissue and translates into a line tracing plot (waveform). The waveform is then used to measure the rate and regularity of heartbeats, as well as the size and position of the chambers, the presence of any damage to the heart, and the effects of drugs or devices used to regulate the heart, such as a pacemaker and take analysis result. The two upper chambers are called atria, and the two lower chambers are called ventricles. A natural electrical system causes the heart muscle to contract and pump blood through the heart to the lungs and the rest of the body.

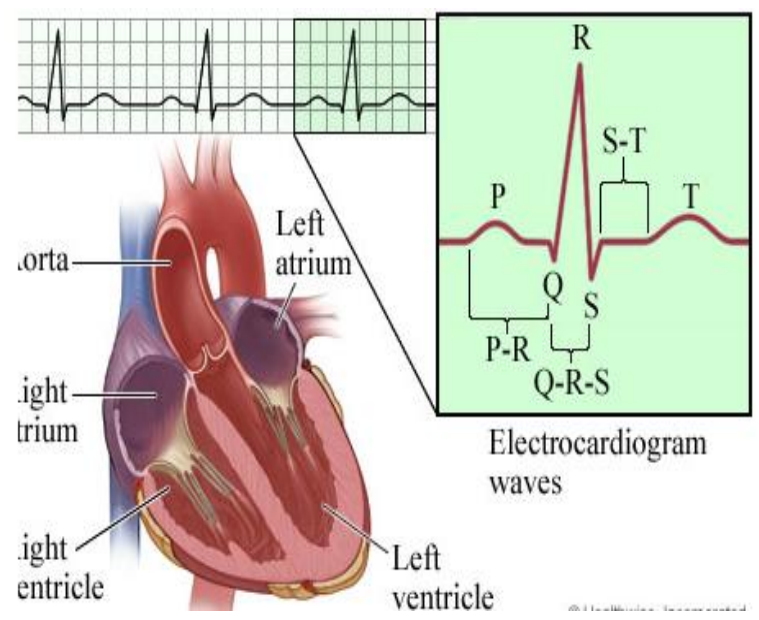

Fig: 2 ECG

\subsection{DWT (Discrete Wavelet Transform)}

Wavelet transform in recent times become highly admired when it comes to study, de-noising, quality optimize and compression of signals and images. The discrete wavelet transform (DWT) is an implementation of the wavelet transform using a discrete set of the wavelet particles and translations obeying few defined rules. In other words, this transform decomposes the signal into mutually orthogonal set of wavelets packet, which is the main difference from the continuous wavelet transform (CWT), or its implementation for the discrete time series sometimes called discrete-time continuous wavelet transform (DT-CWT).

The wavelet can be constructed with the help of scaling function and it defines its properties of scaling. The requirement is that the scaling functions and its discrete transformations must be orthogonal to each other. It implies few mathematical conditions on them which are mentioned everywhere e. g. the dilation equation $\phi(x)=\sum_{k=-\infty}^{\infty} a_{k} \phi(S x-k)$.
In condition (1) Where $S$ called scaling factor (usually chosen as 2). Moreover, the area between the function must be normalized and scaling function must be orthogonal to its integer translates e. $\mathrm{g}$.

$$
\int_{-\infty}^{\infty} \phi(x) \phi(x+l) d x=\delta_{0, l}
$$

After introducing some more conditions (as the restrictions above so does not produce unique solution) we can get results of all these equations, e. g. finite set of coefficients a_k which define the scaling function and also the wavelet. The wavelet is obtained from the scaling function as

$$
\psi(x)=\sum_{k=-\infty}^{\infty}(-1)^{k} a_{N-1-k} \psi(2 x-k)
$$

In condition (3) $\mathrm{N}=$ an even integer. The set of wavelets then forms an orthonormal base which we use in decomposing signal. Note that generally only some of the coefficients a_k are nonzero which simplify the calculations.

$(\mathrm{cA}, \mathrm{cD})=\operatorname{dwt}($ data, wavelet, mode $=$ 'sym' $)$

Parameters: Data - Input signal can be NumPy array, Python list or other iterable object. Both single and double default double precision data format before performing computations.

Wavelet -the wavelist() list or a Wavelet object instance.

Mode - Signal extension mode to deal with the border distortion problem. See MODES for details

\subsection{Methods Of ECG Description Based On WT}

A. State-of-the-Art Single-Lead description of Algorithm Offline

As abovementioned, this work is described and validated in [7] and it is offline WT based ECG.

As such, in that complete description and discrete-time realization of the original dyadic wavelet transform is not reproduced here. For keeping it reserves, it is adequate to state that this delineator performs to detect the ECG waves all characteristic points using a quadratic spline WT, points as onset, peak, and end. It produces the version of the input of $\mathrm{ECH}$ signal and gives five dyadic scales in the range of 21 to 25 . This choice in scale is taken and accepted since signal's energy lies mostly in these scales [7].

In Fig. 2. ECG-like signal has WT at the first five scales. Particular, it was proved that the energy of the QRS complex is lesser in scales high to 24 , and so as to the $\mathrm{P}$ and $\mathrm{T}$ waves have considerable components 25 in scale. These observations are depicted in Fig. 2, which depicts a signal as ECG and the WT's first five scales. Fig. 1 can also be used to show the principle of WT-based ECG delineation. The filtered version's derivative of ECG input signal and the WT at scale $2 \mathrm{k}$ both are proportional to each other with a smoothing function. This smoothed ECG signal has maxima and minima,

and is corresponded by the zero crossings of the WT at different scales and the most of the absolute values of the WT are allied with maximum slopes of that ECG smoothed signal. 
This is demonstrated in Fig. 1, where the waves which are mono phasic like $\mathrm{P}, \mathrm{R}$ and $\mathrm{T}$ produce a pair across the scales with a zero crossing between them, where positive for maximum and negative for minimum. Moreover, when any sharp comes in the input of ECG signal, it is related with the maxima and minima line of scales.

Consequently, the fiducial points of the ECG signal are identified by the WT-based algorithm with the help of information like local maxima, minima and zero crossings.

\section{B. Multilead Delineation}

In experimental practice, on the other hand many leads are obtained at the same time, be it normal 12-lead ECG in conventional experimental settings or the 3-lead arrangement

in rising ambulatory ECG monitoring. In comparison to a single lead delineation, multi lead improves in terms of accuracy, flexibility \& stability to artifacts of the characteristic wave measurement as compared to single -lead by the proper utilization of delineation approaches.

For multi lead delineation, two approaches exist. First one depicts each and every lead individually, to select the "best" describing one there applied a median post processing selection rule over the characteristic wave peaks. Keep the "best" global single-lead delineation [4], [7]. This approach is not yet applicable in case of availability of only two leads and must be changed by a "genie" selection that provides less error when choosing the each point in lead.

\section{CONCLUSION}

To calculate HRV metrics there are many standards adopted and its relevant agreement of its remote application, when it comes to study HRV analysis, there are many conflicting concepts which do not represent a clear consent on HRV metrics objective value. To develop the proper signal processing techniques \& HRV analysis, this proposed work aimed to perform in a proper structure.

This paper proposed an entire relationship between the two compression algorithms i.e. a new CS-based and modern DWT based embedded ECG algorithm. In comparison to a signal adaptive DWT-based counterpart for revamp signal level, non adaptive CS-based compression showed low-grade performance as predicted earlier. Therefore, to apply those techniques, many data sets were employed. In real time mobile application, it makes it fast in computation and so feasible to use the algorithm on it.

\section{REFERENCES}

[1] World Health Organization., "Cardiovascular diseases," 2009. [Online]

[2] M-k Suh, et al. "A Remote Patient Monitoring System for Congestive Heart Failure," JMS, 2011.

[3] H. Mamaghanian, et al. "Compressed Sensing for RealTime Energy-Efficient ECG Compression on Wireless Body Sensor Nodes," IEEE TBE, 2011.

[4] Shimmer Research. (2008). [Online].

[5] R. F. Yazicioglu, et al., "Ultra-low-power wearable biopotential sensor nodes," IEEE EMBS, 2009

[6] R. F. Yazicioglu and et al., "Ultra-low-power wearable biopotential sensor nodes," in Proceedings of IEEE EMBS (EMBC'09), Sep. 2009.
[7] S. M. S. Jalaleddine, C. G. Hutchens, R. D. Stranttan, and W. A. Coberly, "ECG data compression techniques: A unified approach," IEEE Trans. on Biomed. Eng., vol. 37, no. 4, pp. 329-343, 1990.

[8] L. S*ornmo and P. Laguna, Bioelectrical Signal Processing in Cardiac and Neurological Applications. Elsevier Academic Press, 2005.

[9] M. B. Velasco, "Compression of electrocardiograms using cosine modulated filter banks and multi-resolution analysis (in spanish)," Ph.D. dissertation, University of Alcal'a, Spain, Dec. 2004

[10] M. Hilton, "Wavelet and wavelet packets compression of electrocardiogram," IEEE Trans. on Biomed. Eng., vol. 44, no. 5, pp. 394-402, 1997.

[11] Z. Lu, D. Y. Kim, and W. A. Pearlman, "Wavelet compression of ECG signals by the set partitioning in hierarchical trees algorithm," IEEE Trans. On Biomed. Eng., vol. 47, no. 7, pp. 849-856, 2000.

[12] B. A. Rajoub, "An efficient coding algorithm for the compression of ECG signals using the wavelet transform," IEEE Trans. on Biomed. Eng., vol. 49, no. 4, pp. 355-362, 2002

[13] R. Benzid and et al., "Fixed percentage of wavelet coefficients to be zeroed for ECG compression," Electronics Letters, vol. 39, no. 11, pp. 830-831, 2003.

[14] S. Aviyente, "Compressed sensing framework for EEG compression," in Proceedings of the IEEE Workshop on Stat. Signal Proc. (SSP'07), Aug. 2007, pp. 181-184.

[15] S. Ss enay, L. F. Chaparro, M. Sun, and R. J. Sclabassi, "Compressive sensing and random filtering of EEG signals using slepian basis," in Proceedings of the EURASIP EUSIPCO’08, Aug. 2008.

[16] F. Rincon, et. al, "Development and Evaluation of Multilead Wavelet-Based ECG Delineation Algorithms for Embedded Wireless Sensor Nodes," IEEE TITB, 2011.

[17] F. Massien, et al. "Miniaturized wireless ECG monitor for real-time detection of epileptic seizures," $A C M$ TECS, 2013.

[18] AI Maistrou, "Implicit Comparison of Accuracy of Heart Rate Variability Spectral Measures Estimated via Heart Rate and Heart Period Signals," IEEE CinC, 2008.

[19] J. F. Thayer, et al. "A meta-analysis of heart rate variability and neuroimaging studies: Implications for heart rate variability as a marker of stress and health,' NBR, 2012.

[20] W. H. Press, et al, "Fast algorithm for spectral analysis of unevenly sampled data," Astrophysical Journal, 1989.

[21] S-Y Tseng, et al, "An effective heart rate variability processor design based on time-frequency analysis algorithm using windowed Lomb periodogram," IEEE BioCAS, 2010.

[22] K. Kanoun, "A real-time compressed sensing-based personal electrocardiogram monitoring system," IEEE DATE, 2011. 